\title{
4-spin plaquette singlet state in the Shastry-Sutherland compound $\mathrm{SrCu}_{2}\left(\mathrm{BO}_{3}\right)_{2}$
}

\author{
M. E. Zayed ${ }^{1,2,3 \star}$, Ch. Rüegg ${ }^{2,4,5}$, J. Larrea J. ${ }^{1,6}$, A. M. Läuchli ${ }^{7}$, C. Panagopoulos ${ }^{8,9}$, S. S. Saxena ${ }^{8}$, \\ M. Ellerby ${ }^{5}$, D. F. McMorrow ${ }^{5}$, Th. Strässle ${ }^{2}$, S. Klotz ${ }^{10}$, G. Hamel ${ }^{10}$, R. A. Sadykov ${ }^{11,12}$, V. Pomjakushin², \\ M. Boehm ${ }^{13}$, M. Jiménez-Ruiz ${ }^{13}$, A. Schneidewind ${ }^{14}$, E. Pomjakushina ${ }^{15}$, M. Stingaciu ${ }^{15}$, K. Conder ${ }^{15}$ \\ and H. M. Rønnow ${ }^{1}$
}

The study of interacting spin systems is of fundamental importance for modern condensed-matter physics. On frustrated lattices, magnetic exchange interactions cannot be simultaneously satisfied, and often give rise to competing exotic ground states ${ }^{1}$. The frustrated two-dimensional Shastry-Sutherland lattice ${ }^{2}$ realized by $\mathrm{SrCu}_{2}\left(\mathrm{BO}_{3}\right)_{2}$ (refs 3,4) is an important test case for our understanding of quantum magnetism. It was constructed to have an exactly solvable 2-spin dimer singlet ground state within a certain range of exchange parameters and frustration. While the exact dimer state and the antiferromagnetic order at both ends of the phase diagram are well known, the ground state and spin correlations in the intermediate frustration range have been widely debated $^{2,4-14}$. We report here the first experimental identification of the conjectured plaquette singlet intermediate phase in $\mathrm{SrCu}_{2}\left(\mathrm{BO}_{3}\right)_{2}$. It is observed by inelastic neutron scattering after pressure tuning to $\mathbf{2 1 . 5}$ kbar. This gapped singlet state leads to a transition to long-range antiferromagnetic order above $\mathbf{4 0} \mathbf{k b a r}$, consistent with the existence of a deconfined quantum critical point.

In the field of quantum magnetism, geometrically frustrated lattices generally imply major difficulties in analytical and numerical studies. For very few particular topologies, however, it has been shown that the ground state, at least, can be calculated exactly as for the Majumdar-Ghosh model ${ }^{15}$ that solves the $J_{1}-J_{2}$ zigzag chain when $J_{1}=2 J_{2}$. In two dimensions, the ShastrySutherland model $^{2}$ consisting of an orthogonal dimer network of spin $S=1 / 2$ was developed to be exactly solvable. For an interdimer $J^{\prime}$ to intra-dimer $J$ exchange ratio $\alpha \equiv J^{\prime} / J \leq 0.5$ the ground state is a product of singlets on the strong bond $J$. Numerical calculations have further shown that this remains valid up to $\alpha \leq \sim 0.7$ and for small values of three-dimensional (3D) couplings $J^{\prime \prime}$ between dimer layers. At the other end, for $\sim 0.9 \leq \alpha \leq \infty$ the system approaches the well-known $2 \mathrm{D}$ square lattice, which is antiferromagnetically (AFM) ordered, albeit with significant quantum fluctuations that are believed to include resonating singlet correlations resulting in fractional excitations ${ }^{16}$. The phase diagram of the Shastry-Sutherland model, both with and without applied magnetic field, has been intensively studied by numerous theoretical and numerical approaches ${ }^{4}$. In the presence of magnetic field, magnetization plateaus at fractional values of the saturation magnetization corresponding to Mott insulator phases of dimer states, as well as possible superfluid and supersolid phases have been extensively studied ${ }^{7,17-19}$. At zero field, the main unsolved issue is the existence and nature of an intermediate phase for $\sim 0.7 \leq \alpha \leq \sim 0.9$. A variety of quantum phases and transitions between them have been predicted depending on the theoretical technique used: a direct transition from dimer singlet phase to AFM order ${ }^{2,6,7}$, or an intermediate phase with helical order ${ }^{5}$, columnar dimers ${ }^{11}$, valence bond crystal $^{12}$ or resonating valence bond plaquettes ${ }^{9,10}$. Recent results indicate that a plaquette singlet phase is favoured ${ }^{4,20}$. From such a phase, which would have an additional Ising-type order parameter, a subsequent transition to AFM order could provide a realization of the so far elusive deconfined quantum critical point ${ }^{21}$.

The compound strontium copper borate $\mathrm{SrCu}_{2}\left(\mathrm{BO}_{3}\right)_{2}$ is the only known realization of the Shastry-Sutherland model with $S=1 / 2$ spins $^{4}$ and has thus triggered considerable attention in the field of quantum magnetism. The spectrum of $\mathrm{SrCu}_{2}\left(\mathrm{BO}_{3}\right)_{2}$ exhibits an almost dispersionless $\Delta=3 \mathrm{meV}$ gap, and a bound state of two triplets (BT) forms at $E_{\mathrm{BT}} \simeq 5 \mathrm{meV}$. The unusual size and dispersionless nature of the gap is an effect of the frustration that prevents triplets from hopping up to sixth order ${ }^{4}$. The estimated exchange parameters in the material $J \sim 85 \mathrm{~K}$ and $\alpha=0.635$ (ref. 4) or $J \sim 71 \mathrm{~K}$ and $\alpha=0.603$ (ref. 8) place the compound close to an interesting regime $\alpha \sim 0.7$ where correlations may change dramatically at a critical point.

A precious means to tune a quantum magnet across a quantum phase transition is the application of hydrostatic pressure as

\footnotetext{
${ }^{1}$ Laboratory for Quantum Magnetism, Institute of Physics, École Polytechnique Fédérale de Lausanne (EPFL), 1015 Lausanne, Switzerland. ${ }^{2}$ Laboratory for Neutron Scattering and Imaging, Paul Scherrer Institut, 5232 Villigen PSI, Switzerland. ${ }^{3}$ Department of Physics, Carnegie Mellon University in Qatar, Education City, PO Box 24866, Doha, Qatar. ${ }^{4}$ Department of Quantum Matter Physics, University of Geneva, 1211 Geneva 4, Switzerland. ${ }^{5}$ London Centre for Nanotechnology and Department of Physics and Astronomy, University College London, London WC1E 6BT, UK. ${ }^{6}$ Centro Brasileiro de Pesquisas Fisicas, Rua Doutor Xavier Sigaud 150, CEP 2290-180, Rio de Janeiro, Brazil. ${ }^{7}$ Institut für Theoretische Physik, Universität Innsbruck, 6020 Innsbruck, Austria. ${ }^{8}$ Cavendish Laboratory, University of Cambridge, Cambridge CB3 OHE, UK. ${ }^{9}$ Division of Physics and Applied Physics, School of Physical and Mathematical Sciences, Nanyang Technological University, Singapore 637371, Singapore. ${ }^{10}$ IMPMC; CNRS-UMR 7590, Université Pierre et Marie Curie, 75252 Paris, France. ${ }^{11}$ Institute for Nuclear Research, Russian Academy of Sciences, prospekt 60-letiya Oktyabrya 7a, Moscow 117312, Russia. ${ }^{12}$ Vereshchagin Institute for High Pressure Physics, Russian Academy of Sciences, 108840, Moscow, Troitsk, Russia. ${ }^{13}$ Institut Laue-Langevin, 71 avenue des Martyrs - CS 20156 38042 Grenoble Cedex 9, France. ${ }^{14}$ Jülich Centre for Neutron Science (JCNS) at Heinz Maier-Leibnitz Zentrum (MLZ), Forschungszentrum Jülich GmbH, Lichtenbergstr. 1, 85748 Garching, Germany. ${ }^{15}$ Laboratory for Scientific Developments and Novel Materials, Paul Scherrer Institut, 5232 Villigen PSI, Switzerland. *e-mail: mohamed.zayed@epfl.ch
} 


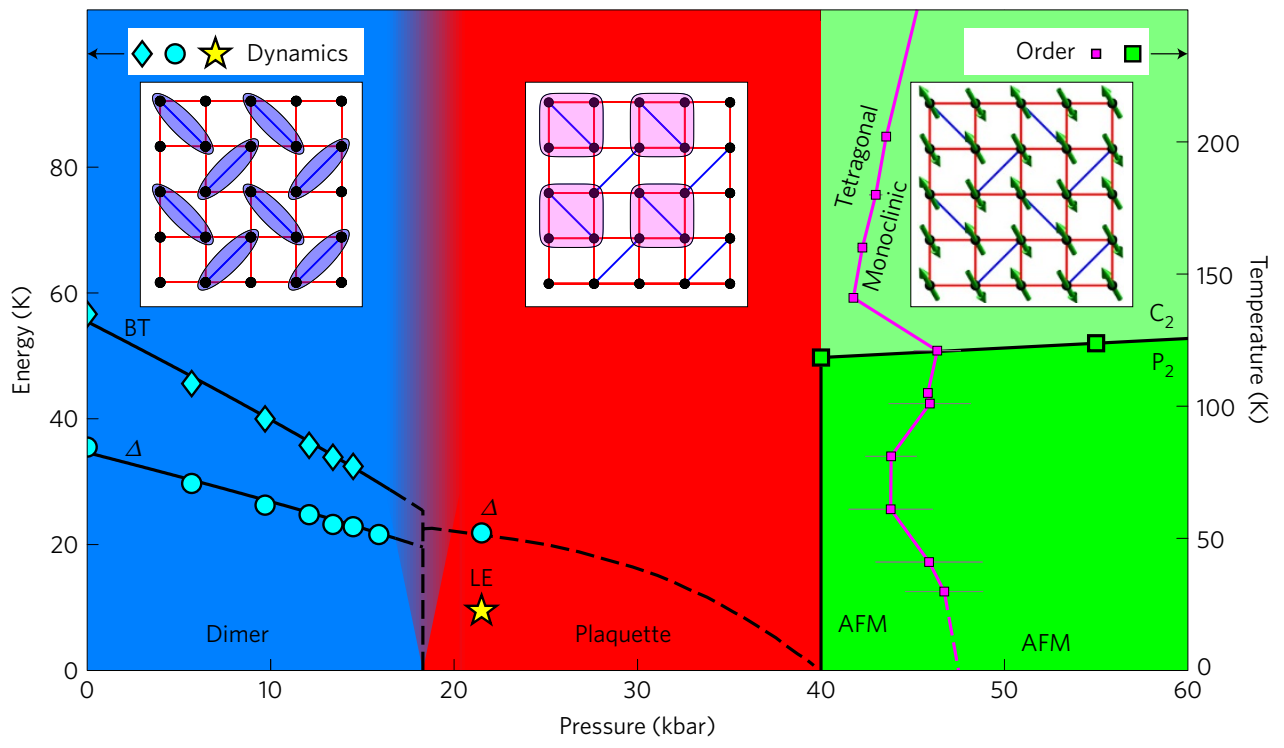

Figure 1 | Phase diagram of $\mathrm{SrCu}_{2}\left(\mathrm{BO}_{3}\right)_{2}$ as a function of pressure and temperature, including excitation energies. The blue region is the dimer phase, the red region the newly identified plaquette phase, and the green region is the antiferromagnetic phase where $Q=(1,0,0)$ magnetic Bragg peaks, indicated by green squares, are observed only above $40 \mathrm{kbar}$. Circles are the triplet gap energy $\Delta$ at $Q=(2,0, L)$, diamonds are the corresponding two-triplet bound state (BT) energy $E_{\mathrm{B} T}$ and the star is a new low-energy excitation $(\mathrm{LE})$ observed at $Q=(1,0,1)$. The magenta line shows the tetragonal to monoclinic structural transition. The procedure used to obtain it and its error bars is described in ref. 28. The corresponding monoclinic space groups are indicated ${ }^{29,30}$. The dashed line in the plaquette phase is the extrapolated energy gap using ref. 9. The insets depict the corresponding ground states. All of the experimental points are from this study.

it directly modifies the atomic distances and bridging angles, such as $\mathrm{Cu}-\mathrm{O}-\mathrm{Cu}$ and thus the magnetic exchange integrals. Quantum phase transitions were successfully discovered in dimer magnets following application of pressure ${ }^{22}$. However highpressure measurements remain technically challenging. In the case of $\mathrm{SrCu}_{2}\left(\mathrm{BO}_{3}\right)_{2}$, magnetic susceptibility ${ }^{23}$ and electron spin resonance ${ }^{24}$ to moderate pressures $(p \leq 12 \mathrm{kbar})$ indicate a softening of the gap, while the combined effect of pressure and field was measured by susceptibility and $\mathrm{NMR}^{25}$. In the latter case, magnetic order occurring at $24 \mathrm{kbar}$ and $7 \mathrm{~T}$ on a fraction of the dimers was proposed. In an X-ray diffraction investigation, the temperature dependence of the lattice parameters was analysed as an indirect proxy for the singlet-triplet gap leading to the suggestion that it closes at $20 \mathrm{kbar}^{26}$. At even higher pressures, neutron and X-ray diffraction experiments observed a transition above $45 \mathrm{kbar}$ from the ambient I42M tetragonal space group to monoclinic ${ }^{27-30}$.

Here we present neutron spectroscopy results, which directly determine the pressure dependence of the gap and through the dynamic structure factor allow us to address the nature of the correlations. Figure 1 summarizes the phase diagram of $\mathrm{SrCu}_{2}\left(\mathrm{BO}_{3}\right)_{2}$, which we determined in this study. The exact dimer phase survives up to $16 \mathrm{kbar}$. The gap decreases from $3 \mathrm{meV}$ to $2 \mathrm{meV}$, but does not vanish. At $21.5 \mathrm{kbar}$, we discover experimentally a new, intermediate phase. We identify it by its inelastic neutron scattering spectrum as the formation of 4-spin plaquette singlets. Above $40 \mathrm{kbar}$ and below $117 \mathrm{~K}$ we find by neutron diffraction that AFM order appears (Supplementary Fig. 6) while the compound probably still has tetragonal symmetry with orthogonal dimers. Above $\sim 45 \mathrm{kbar}$, a structural distortion takes place and the symmetry becomes monoclinic, implying nonorthogonal dimers ${ }^{28,29} \cdot \mathrm{SrCu}_{2}\left(\mathrm{BO}_{3}\right)_{2}$ is magnetically ordered after the distortion, but can no longer be described appropriately by the original Shastry-Sutherland model. The transition from 2-spin dimer to 4-spin plaquette singlets appears to be of first order, whereas the transition from the plaquette to the AFM phase could be of second order and concomitant with the continuous closure of the plaquette gap as sketched in Fig. 1 or of first order ${ }^{9,20}$.
To allow a quantitative comparison to theoretical predictions, we establish the pressure dependence of the exchange parameters $J_{\chi}(p), J_{\chi}^{\prime}(p)$ and $\alpha(p)$ by measuring magnetic susceptibility $\chi(p, T)$ and fitting it using 20 -site exact diagonalization. The peak in susceptibility shifts to lower temperature as pressure increases up to $10 \mathrm{kbar}$ (Fig. 2a). This suggests a reduction of the spin gap. We parametrize the pressure dependence of $J$ and $J^{\prime}$ by linear fits (Fig. 2b). $J$ has the larger slope so that $\alpha$ increases with pressure. Having established $\alpha(p)$ we see that the critical pressure lying between $16 \mathrm{kbar}$ and $21.5 \mathrm{kbar}$ corresponds to $0.66<\alpha_{\mathrm{c}}<0.68$, in good agreement with theoretical predictions ${ }^{4,12,20}$.

A selection from the neutron spectra leading to the phase diagram is presented in Fig. 3; additional spectra at various momenta transfer $Q$ are shown in the Supplementary Information. Up to $16 \mathrm{kbar}$ an essentially $\mathrm{Q}$-independent linear decrease of the gap energy is observed (Figs 1 and 3a). The measurement of the dispersion and of the structure factor in that pressure range shows that the spin system is still in its original 'exact dimer' phase. The gap value and the integrated intensity decrease linearly with pressure. The dispersion increases slightly with pressure, which can be understood by the increase of $\alpha$ (ref. 6). Interestingly, the bound triplet energy $E_{\mathrm{BT}}$ softens twice as fast, implying that the triplet binding energy, $\delta=2 \Delta-E_{\mathrm{BT}}=1.19$ (2) $\mathrm{meV}$, remains quasi pressure independent. This results in the unusual situation that extrapolating the softenings, the bound triplet would reach zero energy before the single triplet, and hence that, before that point, exciting a bound state of two triplets would cost less energy than exciting one triplet.

$\mathrm{SrCu}_{2}\left(\mathrm{BO}_{3}\right)_{2}$ enters a new quantum phase between 16 and $21.5 \mathrm{kbar}$, where a discontinuity in the gap softening occurs. The inelastic neutron scattering peaks corresponding to the gap energy, $\Delta \simeq 2 \mathrm{meV}$, at these two pressures remain unchanged (Fig. $3 \mathrm{~b}$ ). The discontinuity is also visible in the intensities (Fig. 3d), where the linear decrease with pressure exhibits an abrupt halt above $16 \mathrm{kbar}$.

The transition to a new quantum phase is further asserted by a new type of excitation suddenly appearing at the higher pressure (Fig. 3b,c). We label this new low-energy excitation LE. LE is clearly 

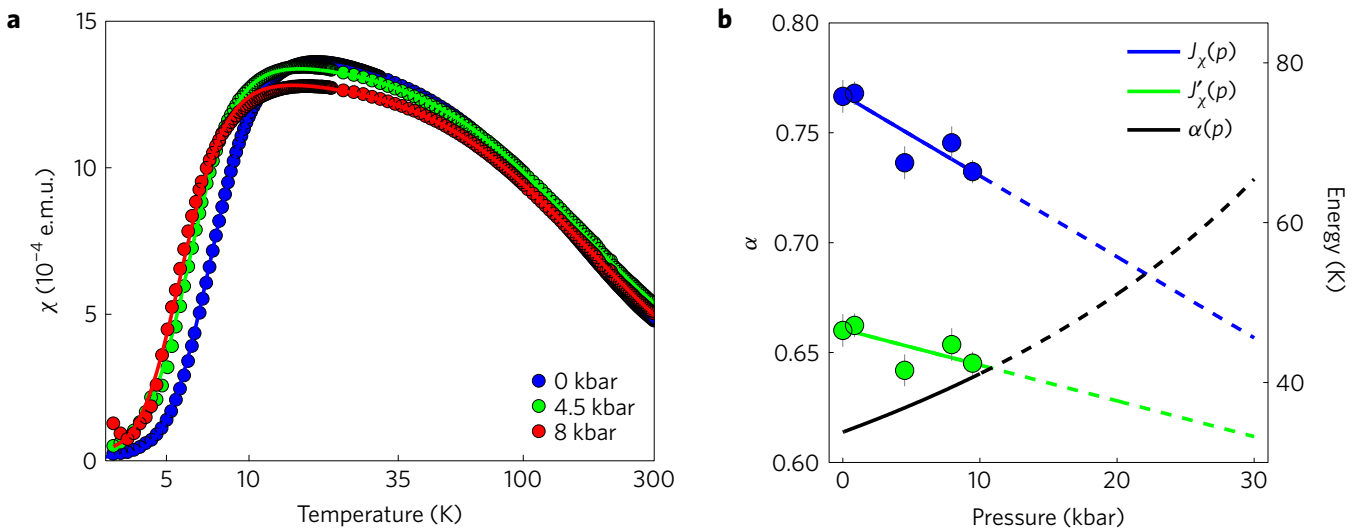

Figure $\mathbf{2}$ | Pressure dependence of the magnetic susceptibility and of the exchange parameters in $\mathrm{SrCu}_{2}\left(\mathrm{BO}_{3}\right)_{2}$. a, Magnetic susceptibility at three pressures below $10 \mathrm{kbar}$ with fits to calculations by exact diagonalization (solid lines), $H=0.5 \mathrm{~T}$. b. Extracted exchange parameters $J_{\chi}(p)$ and $J_{\chi}^{\prime}(p)$ with linear fits and their ratio $\alpha(p)$. The error bars in $\mathbf{b}$ represent standard deviation of the fit.

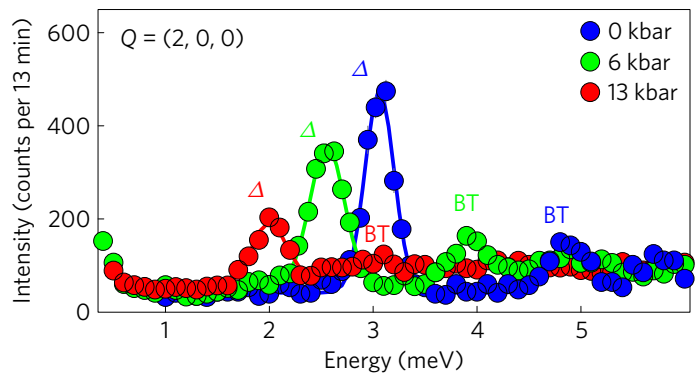

b

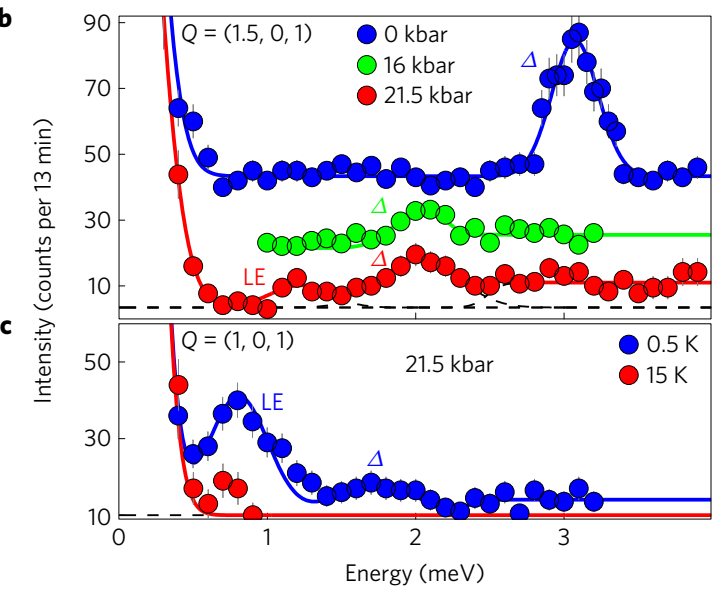

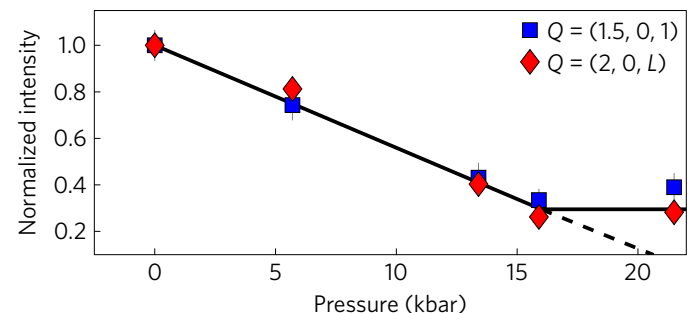

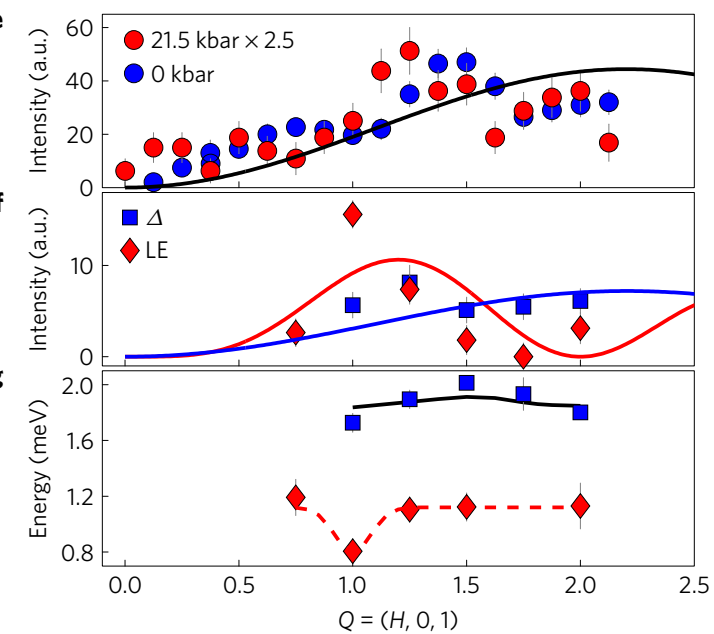

Figure 3 | Inelastic neutron scattering measurements of $\mathrm{SrCu}_{2}\left(\mathrm{BO}_{3}\right)_{2}$ under hydrostatic pressure. a, Energy spectra with triplet gap $\Delta$ and two-triplet bound-state BT energies softening in the dimer phase (Set-up 1). b. Discontinuity in the gap softening between 16 and $21.5 \mathrm{kbar}$ (Set-up 2). c, New low-energy excitation $L E$ at $Q=(1,0,1)$ (Set-up 3). d, Pressure dependence of the gap integrated intensity (Set-ups 1-3). e, Momentum dependence of the intensity at the gap energy $\Delta$, background subtracted (Set-up 2). The black line is the isolated dimer structure factor. $\mathbf{f}$, Intensities of $\Delta$ and LE at $21.5 \mathrm{kbar}$. The red (blue) line is the full plaquette T1 (T2) structure factor (see Fig. 4). g, Dispersion of $\Delta$ and LE at $21.5 \mathrm{kbar}$. The black line is a scaled ambient pressure dispersion and the red dashed line is a guide to the eye. Error bars for inelastic neutron scattering intensities (a,b,c,e) are obtained from the square root of the number of counts assuming a Poisson distribution. The error bars in $\mathbf{d}, \mathbf{f}, \mathbf{g}$ are standard deviations of the fit.

visible around $1 \mathrm{meV}$ for $Q=(1,0,1),(-1,0,1)$ and $(1,0,1.5)$ at $0.5 \mathrm{~K}$ and is not observed at $15 \mathrm{~K}$, which proves its magnetic origin.

At $21.5 \mathrm{kbar}$, beyond the discontinuity, the $2 \mathrm{meV}$ excitation displays a remarkable similarity to the ambient-pressure $\Delta$ as shown by constant energy scans along $Q=(H, 0,1)$ in Fig. $3 \mathrm{e}$. Both qualitatively follow the isolated dimer structure factor. This is further confirmed by extracting the structure factors from energy scans (Fig. 3f) and by comparing the dispersion to the ambient pressure dispersion (Fig. 3g). We therefore keep labelling this excitation $\Delta$. LE on the other hand is more dispersive, $\sim 0.4 \mathrm{meV}$ in the measured momentum range, and has a different structure factor strongly peaking between $Q=(1,0,1)$ and $Q=(1.25,0,1)$. This behaviour is reminiscent of a 4 -spin plaquette structure factor (red line in Fig. 3f) that is further discussed in Fig. 4.

To interpret the new excitation and the observed momentum dependence of the dynamical structure factors, it is illustrative to consider the simplified case of an isolated 4-spin plaquette, described in the Methods, which has a singlet ground state and shows two low-lying excitations $\mathrm{T}_{1}$ and $\mathrm{T}_{2}$. The structure factors of these excitations, summed over the two possible 'full' plaquette orientations (Fig. 4a), are shown in Fig. 4b,c together with those of a 'void' plaquette (Fig. $4 \mathrm{~d}-\mathrm{f}$ ) containing no diagonal bond. $\mathrm{T}_{1}$ 
a

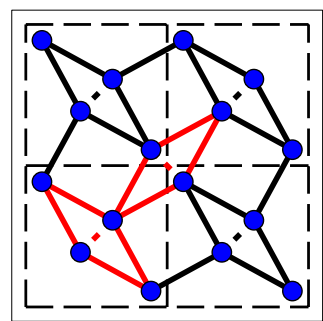

d

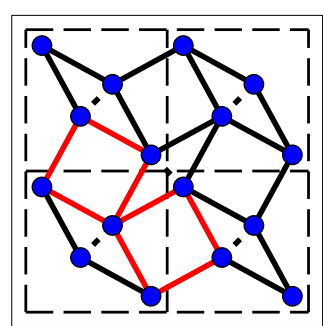

b

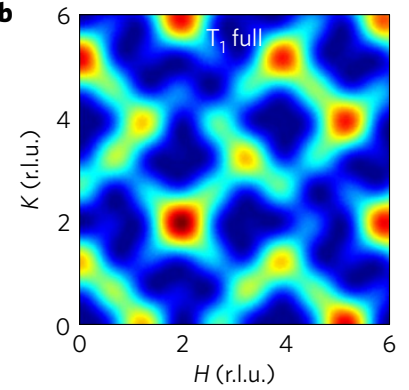

e

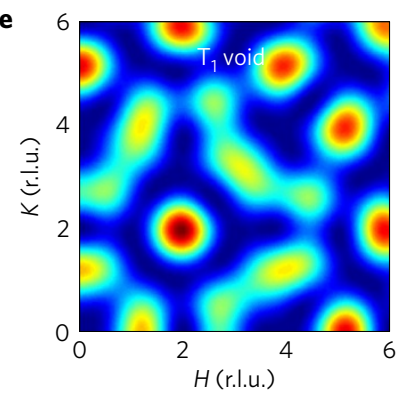

C

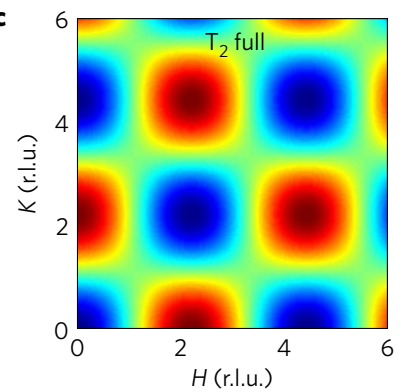

f

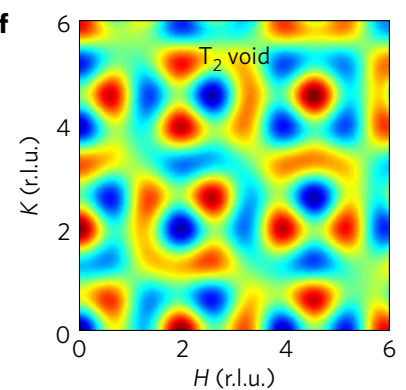

g

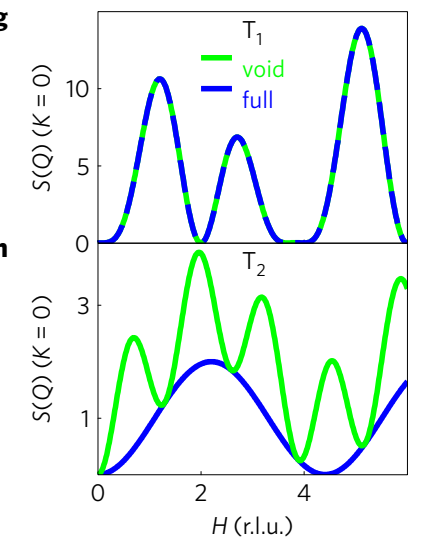

i

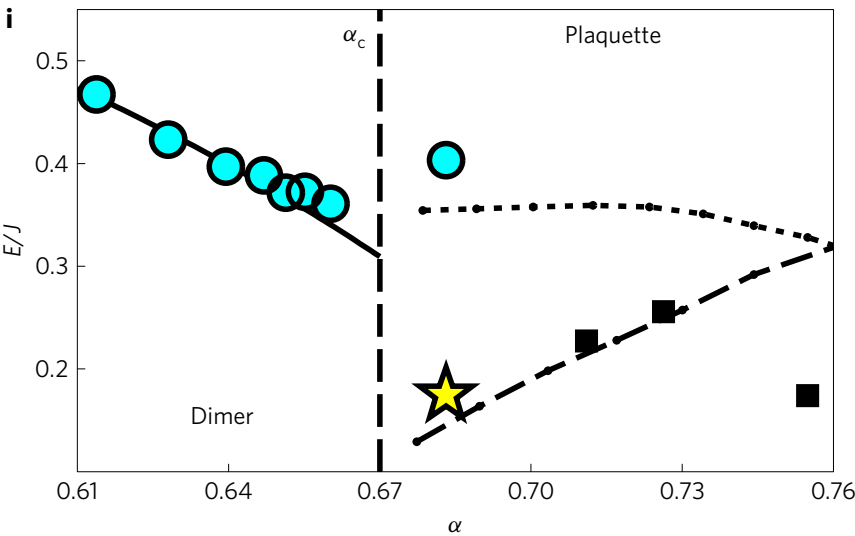

Figure 4 | Plaquette phase in $\mathrm{SrCu}_{2}\left(\mathrm{BO}_{3}\right)_{2}$. a, Full plaquettes containing a diagonal bound. b,c, Full plaquette structure factors for excitations $\mathrm{T}_{1}(\mathbf{b})$ and $\mathrm{T}_{2}$ (c); $\mathbf{c}$ is identical to the structure factor of two orthogonal isolated dimers. $\mathbf{d}$, Void plaquettes. $\mathbf{e}, \mathbf{f}$, Void plaquette structure factors for excitations $T_{1}$ (e) and $T_{2}(\mathbf{f})$. In each case the structure factors $S^{x x}+S^{y y}+S^{z z}$ are calculated as the sum over the two possible full (a) or two possible void (d) plaquette orientations in the $\mathrm{SrCu}_{2}\left(\mathrm{BO}_{3}\right)_{2}$ geometry. $\mathbf{g}$, Structure factor along $Q_{h k}=\left(Q_{h}, Q_{k}\right)=(H, 0)$ for $T_{1}$; void plaquette in green and full plaquette in blue are identical. $\mathbf{h}$, Structure factor along $Q_{h k}=\left(Q_{h}, Q_{k}\right)=(H, 0)$ for $T_{2}$; void plaquette in green and full plaquette in blue. The blue line is also the isolated dimer structure factor. $\mathbf{i}$, Excitation energies as a function of $\alpha(p)$ extrapolated from Fig. 2b. Comparison between experiment (same points as in Fig. 1) and theoretical predictions: dimer gap energy adapted from ref. 11 (solid line), low- and high-energy triplet excitations in the plaquette phase from ref. 10 (dotted lines), and for columnar plaquette block energies ref. 13 (black squares).

has a structure factor peaking between $Q_{h k}=\left(Q_{h}, Q_{k}\right)=(1,0)$ and $Q_{h k}=(1.25,0)$ in the $2 \mathrm{D}$ geometry of $\mathrm{SrCu}_{2}\left(\mathrm{BO}_{3}\right)_{2}$ for both full and void plaquettes (Fig. 4g). $\mathrm{T}_{2}$, however, has a structure factor identical to that of an isolated dimer on the diagonal bond only for the full plaquette (Fig. 4c,f,h). While an extended many-body calculation would be needed for a fully quantitative comparison, the isolated plaquette considered here displays the main characteristics of the new intermediate pressure phase: a non-magnetic gapped ground state; a low-energy triplet (LE) with structure factor peaking above $Q_{h k}=(1,0)$; and another low-energy excitation $(\Delta)$ with structure factor identical to the singlet-triplet transition in the exact dimer phase. We thus identify the discovered phase as composed of 4spin plaquette singlets, with excitation LE corresponding to $T_{1}$ and excitation $\Delta$ corresponding to $\mathrm{T}_{2}$. Comparing the experimental intensities to this simple calculation favours the singlets sitting on 'full' plaquettes containing diagonal bonds, but calculations of the structure factor for the extended model are required for verification of this point.
To analyse further the interacting plaquette system, we plot in Fig. $4 \mathrm{i}$ the measured energies $E / J$ versus $\alpha$, which enables a direct comparison between our results and the calculations for the low- and high-energy resonating valence bond-like plaquette excitations ${ }^{10}$, and columnar plaquette block energy ${ }^{13}$. Experimental and calculated ${ }^{6}$ gap energies in the dimer phase are in excellent agreement. Beyond the transition, there is qualitative agreement for the energy scales; in particular, the observed energies of LE and of $\Delta$ for $21.5 \mathrm{kbar}$ are close to the expected low- and high-energy plaquette excitations of ref. 10 for $\alpha=0.68$.

Our results can also explain the occurrence of magnetic ordering proposed by NMR measurements at $24 \mathrm{kbar}$ and $7 \mathrm{~T}$ (ref. 25): the new spin $S=1$ excitation LE being low in energy $(0.8 \mathrm{meV})$, a $7 \mathrm{~T}$ field is sufficient to close the related gap and to obtain a magnetic ground state. This field-induced quantum critical point and resulting phase will be related to the field-induced BEC physics observed in dimer singlet systems ${ }^{31}$, but could reveal new phenomena due to the strong frustration in the Shastry-Sutherland 
model. In particular, the evolution of the magnetization plateaus in $\mathrm{SrCu}_{2}\left(\mathrm{BO}_{3}\right)_{2}$ with pressure remains to be studied. On the basis of the results presented here, we can predict that, in particular, the pressure range between 15 and $25 \mathrm{kbar}$ will be of high interest.

In conclusion, we have performed high-pressure experiments on $\mathrm{SrCu}_{2}\left(\mathrm{BO}_{3}\right)_{2}$ and tuned the compound to experimentally identify a novel singlet phase consistent with the conjectured plaquette state at intermediate exchange ratio in the Shastry-Sutherland lattice. We observed a first-order transition taking place between two magnetically disordered states: the exact 2-spin dimer singlet and the 4 -spin plaquette singlet phase. The dominant correlations in the plaquette phase involve a four-spin unit and are characterized by a low-lying triplet excitation that is not present in the dimer phase and that gives access to new types of field- and pressure-induced quantum critical points. The plaquette phase itself is suppressed at higher pressures where classical Néel order is found. Particularly exciting is the fact that the existence of two possible plaquette singlet coverings offers an Ising-type order parameter. This may turn the transition from plaquette to Néel phase into a deconfined quantum critical point at $40 \mathrm{kbar}$.

During the review of this manuscript, a new publication ${ }^{32}$ came to our attention, where high field magnetization measurements confirm the existence of a novel phase at $22 \mathrm{kbar}$, and its implication on the magnetization plateaus of $\mathrm{SrCu}_{2}\left(\mathrm{BO}_{3}\right)_{2}$ is discussed.

\section{Methods}

Methods, including statements of data availability and any associated accession codes and references, are available in the online version of this paper.

\section{Received 21 February 2016; accepted 25 May 2017;} published online 17 July 2017

\section{References}

1. Lhuillier, C. \& Misguich, G. in High Magnetic Fields (eds Berthier, C., Lvy, L. \& Martinez, G.) 161-190 (Lecture Notes in Physics, 595, Springer, 2001).

2. Shastry, B. S. \& Sutherland, B. Exact ground state of a quantum mechanical antiferromagnet. Physica B+C 108, 1069-1070 (1981).

3. Kageyama, H. et al. Exact dimer ground state and quantized magnetization plateaus in the two-dimensional spin system $\mathrm{SrCu}_{2}\left(\mathrm{BO}_{3}\right)_{2}$. Phys. Rev. Lett. 82 3168-3171 (1999).

4. Miyahara, S. \& Ueda, K. Theory of the orthogonal dimer Heisenberg spin model for $\mathrm{SrCu}_{2}\left(\mathrm{BO}_{3}\right)_{2}$. J. Phys. Condens. Matter 15, R327 (2003).

5. Albrecht, M. \& Mila, F. First-order transition between magnetic order and valence bond order in a 2D frustrated Heisenberg model. Europhys. Lett. 34, 145-150 (1996)

6. Weihong, Z., Hamer, C. J. \& Oitmaa, J. Series expansions for a Heisenberg antiferromagnetic model for $\mathrm{SrCu}_{2}\left(\mathrm{BO}_{3}\right)_{2}$. Phys. Rev. B 60, 6608-6616 (1999).

7. Müller-Hartmann, E., Singh, R. R. P., Knetter, C. \& Uhrig, G. S. Exact demonstration of magnetization plateaus and first-order Dimer-Néel phase transitions in a modified Shastry-Sutherland model for $\mathrm{SrCu}_{2}\left(\mathrm{BO}_{3}\right)_{2}$ Phys. Rev. Lett. 84, 1808-1811 (2000).

8. Knetter, C., Bühler, A., Müller-Hartmann, E. \& Uhrig, G. S. Dispersion and symmetry of bound states in the Shastry-Sutherland model. Phys. Rev. Lett. 85, 3958-3961 (2000).

9. Koga, A. \& Kawakami, N. Quantum phase transitions in the ShastrySutherland model for $\mathrm{SrCu}_{2}\left(\mathrm{BO}_{3}\right)_{2}$. Phys. Rev. Lett. 84, 4461-4464 (2000).

10. Takushima, Y., Koga, A. \& Kawakami, N. Competing spin-gap phases in a frustrated quantum spin system in two dimensions. J. Phys. Soc. Jpn 70, 1369-1374 (2001).

11. Zheng, W., Oitmaa, J. \& Hamer, C. J. Phase diagram of the Shastry-Sutherland antiferromagnet. Phys. Rev. B 65, 014408 (2001).

12. Läuchli, A., Wessel, S. \& Sigrist, M. Phase diagram of the quadrumerized Shastry-Sutherland model. Phys. Rev. B 66, 014401 (2002)

13. Al Hajj, M. \& Malrieu, J.-P. Phase transitions in the Shastry-Sutherland lattice. Phys. Rev. B 72, 094436 (2005).

14. Ronquillo, D. C. \& Peterson, M. R. Identifying topological order in the Shastry-Sutherland model via entanglement entropy. Phys. Rev. B 90, 201108(R) (2014)
15. Majumdar, C. K. \& Ghosh, D. K. On next nearest neighbor interaction in linear chain. I. J. Math. Phys. 10, 1388-1398 (1969).

16. Dalla Piazza, B. et al. Fractional excitations in the square-lattice quantum antiferromagnet. Nat. Phys. 11, 62-68 (2015).

17. Momoi, T. \& Totsuka, K. Magnetization plateaus of the Shastry-Sutherland model for $\mathrm{SrCu}_{2}\left(\mathrm{BO}_{3}\right)_{2}$ : spin-density wave, supersolid, and bound states. Phys. Rev. B 62, 15067-15078 (2000).

18. Dorier, J., Schmidt, K. P. \& Mila, F. Theory of magnetization plateaux in the Shastry-Sutherland model. Phys. Rev. Lett. 101, 250402 (2008).

19. Matsuda, Y. H. et al. Magnetization of $\mathrm{SrCu}_{2}\left(\mathrm{BO}_{3}\right)_{2}$ in Ultrahigh Magnetic Fields up to 118 T. Phys. Rev. Lett. 111, 137204 (2013).

20. Corboz, P. \& Mila, F. Tensor network study of the Shastry-Sutherland model in zero magnetic field. Phys. Rev. B 87, 115144 (2013).

21. Senthil, T., Vishwanath, A., Balents, L., Sachdev, S. \& Fisher, M. P. A. Deconfined quantum critical points. Science 303, 1490-1494 (2004).

22. Merchant, P. et al. Quantum and classical criticality in a dimerized quantum antiferromagnet. Nat. Phys. 10, 373-379 (2014).

23. Kageyama, H., Mushnikov, N. V., Yamada, M., Goto, T. \& Ueda, Y. Quantum phase transitions in the orthogonal dimer system $\mathrm{SrCu}_{2}\left(\mathrm{BO}_{3}\right)_{2}$. Physica $B$ 329-333, 1020-1023 (2003).

24. Sakurai, T. et al. High-field and high-pressure ESR measurements of $\mathrm{SrCu}_{2}\left(\mathrm{BO}_{3}\right)_{2}$. J. Phys. Conf. Ser. 150, 042171 (2009).

25. Waki, T. et al. A novel ordered phase in $\mathrm{SrCu}_{2}\left(\mathrm{BO}_{3}\right)_{2}$ under high pressure. J. Phys. Soc. Jpn. 76, 073710 (2007).

26. Haravifard, S. et al. Continuous and discontinuous quantum phase transitions in a model two-dimensional magnet. Proc. Natl Acad. Sci. USA 109, 2286-2289 (2012)

27. Loa, I. et al. Crystal structure and lattice dynamics of at high pressures. Physica B 359-361, 980-982 (2005).

28. Zayed, M. E. et al. Temperature dependence of the pressure induced monoclinic distortion in the spin Shastry-Sutherland compound $\mathrm{SrCu}_{2}\left(\mathrm{BO}_{3}\right)_{2}$ Solid State Commun. 186, 13-17 (2014).

29. Haravifard, S. et al. Emergence of long-range order in sheets of magnetic dimers. Proc. Natl Acad. Sci. USA 111, 14372-14377 (2014).

30. Zayed, M. Novel States in Magnetic Materials under Extreme Conditions. A High Pressure Neutron Scattering Study of the Shastry-Sutherland Compound $\mathrm{SrCu}_{2}$ $\left(\mathrm{BO}_{3}\right)_{2} \mathrm{PhD}$ thesis, ETH Zurich (2010)

31. Ruegg, C. et al. Bose-Einstein condensation of the triplet states in the magnetic insulator $\mathrm{TlCuCl}_{3}$. Nature 423, 62-65 (2003).

32. Haravifard, S. et al. Crystallization of spin superlattices with pressure and field in the layered magnet $\mathrm{SrCu}_{2}\left(\mathrm{BO}_{3}\right)_{2}$. Nat. Commun. 7, 11956 (2016).

\section{Acknowledgements}

We thank A. Magee for her contributions to the susceptibility measurements, M. Merlin and M. Hanfland for support during high-pressure X-ray diffraction experiments at the ESRF, and M. Ay and P. Link for assistance during neutron scattering experiments. We acknowledge F. Mila and B. Normand for many useful discussions. We also thank CamCool Research Ltd for supplying the pressure cells for the SQUID measurements. This work is based on experiments performed at the Swiss spallation neutron source SINQ, Paul Scherrer Institute, Villigen, Switzerland, at the FRM-2, Munich, Germany, and at the ILL, Grenoble, France. We thank the Swiss National Science Foundation SNF and the Royal Society (UK) for financial support. The work in London and Cambridge was supported by the EPSRC. J.L.J. acknowledges the Science Without Borders program of $\mathrm{CNPq} / \mathrm{MCTI}-B r a z i l$ and C.P. acknowledges financial support from the National Research Foundation (NRF) of Singapore, through NRF Investigatorship (Reference No. NRF-NRFI2015-04).

\section{Author contributions}

M.E.Z., C.R. and H.M.R. designed the research, performed the experiments and analysed the data. A.M.L. computed the magnetic susceptibility by exact diagonalization. C.P. S.S.S. and M.E. helped with susceptibility experiments. T.S., S.K., G.H. and R.A.S provided neutron high-pressure techniques. M.B., M.J.-R., A.S., V.P. and T.S. provided support for neutron experiments. E.P., M.S. and K.C. synthesized the $\mathrm{SrCu}_{2}\left(\mathrm{BO}_{3}\right)_{2}$ samples. J.L.J. and D.F.M. contributed to interpretation of the data. M.E.Z., C.R. and H.M.R. wrote the manuscript with contributions from all co-authors.

\section{Additional information}

Supplementary information is available in the online version of the paper. Reprints and permissions information is available online at www.nature.com/reprints. Publisher's note: Springer Nature remains neutral with regard to jurisdictional claims in published maps and institutional affiliations. Correspondence and requests for materials should be addressed to M.E.Z

\section{Competing financial interests}

The authors declare no competing financial interests. 


\section{Methods}

Experiments. Inelastic neutron scattering data were collected on three instruments: IN14 at ILL, TASP at SINQ-PSI and PANDA at FRM-2 (ref. 33). Piston-cylinder pressure cells based on hard $\mathrm{Al}$ alloy and hard steel allowed for a single-crystal sample mass of $3 \mathrm{~g}$ below $16 \mathrm{kbar}$ (ref. 34). The 16 and $21.5 \mathrm{kbar}$ pressures were reached with a McWhan ${ }^{34}$ pressure cell and a sample mass of $0.2 \mathrm{~g}$ At $21.5 \mathrm{kbar}$, the sample was cooled down to both $2 \mathrm{~K}$ and $0.5 \mathrm{~K}$ to account for a possible unusual finite temperature damping ${ }^{35}$. AFM ordering was investigated by neutron diffraction on IN8 at ILL, with an opposed anvils Paris-Edinburgh press $^{34,36}$ and a sample mass of about $0.1 \mathrm{~g}$. Synchrotron X-ray diffraction was performed on ID9a at ESRF with a diamond anvil cell and microgram samples ${ }^{28}$. The details of the set-ups used with corresponding crystal orientations are given in Supplementary Table 1.

The pressure dependence of magnetic susceptibility was measured on a MPMS SQUID magnetometer (Quantum Design) using non-magnetic CuBe clamp pressure cells (CamCell) and pressure was calibrated by the superconducting transition of $\mathrm{Pb}$

Data analysis. The pressure-dependent gap $\Delta\left(J_{x}(p), J_{x}^{\prime}(p)\right)$ obtained through the $Q=0$ expansion of ref. 11 with exchange parameters from fits to susceptibility data is in good agreement with the direct inelastic neutron scattering gap measurement $\Delta_{Q}(p)$. To take into account the small Q-dependence of $\Delta_{Q}$, due to Dzyaloshinskii-Moriya interactions ${ }^{37}$, we additionally used $\Delta_{Q}(p)=\Delta\left(J_{\chi}(p), J_{\chi}^{\prime}(p)\right)+D_{Q}(p)$, where the dispersion of $D_{Q}$ is of the order of $0.2 \mathrm{meV}$.

The 4-spin plaquette is described by the Hamiltonian:

$$
\mathcal{H}=J^{\prime}\left(\mathbf{S}_{1} \mathbf{S}_{2}+\mathbf{S}_{2} \mathbf{S}_{3}+\mathbf{S}_{3} \mathbf{S}_{4}+\mathbf{S}_{1} \mathbf{S}_{4}\right)+J\left(\mathbf{S}_{1} \mathbf{S}_{3}\right),
$$

where the last term represents a diagonal bond between sites 1 and 3 (a 'full' plaquette), and should be removed for a 'void' plaquette without such a diagonal bond. The eigenstates of $\mathcal{H}$ can be separated over two sectors depending on the value of the quantum number $S_{1,3}$ for the spins $S_{1}+S_{3}$ on the diagonal bond and $S_{2,4}$ for the spins $\mathbf{S}_{2}+\mathbf{S}_{4}$ on the outer sites ${ }^{30,38}$. A study of the excitation spectrum of such a plaquette shows that for $\alpha \geq 0.5$ the ground state is an $S=0$ singlet of four spins. Two low-lying excitations $\mathrm{T}_{1}$ and $\mathrm{T}_{2}$ are present. For $\alpha \geq 1, \mathrm{~T}_{1}$ has the lower energy, while for $0.5 \geq \alpha \geq 1 \mathrm{~T}_{2}$ does. $\mathrm{T}_{1}$ corresponds to a triplet excitation with both $\mathrm{S}_{1,3}$ and $\mathrm{S}_{2,4}$ equal to 1 . In the full plaquette, $\mathrm{T}_{2}$ is four-fold degenerate and corresponds to a singlet on the diagonal $S_{1,3}=0$ plus two free spins, $S_{2,4}=0$ or 1 . The corresponding structure factor is identical to that of the singlet-triplet excitation on the isolated diagonal bond. For the void plaquette, $\mathrm{T}_{2}$ is seven-fold degenerate and the structure factor does not match the isolated dimer. We note that, in general, the maxima and minima of the isolated models structure factors are not commensurate with the reciprocal lattice.

Data availability. The data that support the plots within this paper and other findings of this study are available from the corresponding author on request.

\section{References}

33. Schneidewind, A. \& Čermàk, P. PANDA: Cold three axes spectrometer. J. Large-Scale Res. Facil. 1, A12 (2015).

34. Klotz, S. Techniques in High Pressure Neutron Scattering (CRC Press, Taylor and Francis, 2013).

35. Zayed, M. E. et al. Correlated decay of triplet excitations in the Shastry-Sutherland compound $\mathrm{SrCu}_{2}\left(\mathrm{BO}_{3}\right)_{2}$. Phys. Rev. Lett. 113, 067201 (2014)

36. Klotz, S. Phonon dispersion curves by inelastic neutron scattering to $12 \mathrm{GPa}$. Z. Kristallogr. 216, 420-429 (2001).

37. Kakurai, K. et al. Neutron scattering investigation on quantum spin system $\mathrm{SrCu}_{2}\left(\mathrm{BO}_{3}\right)_{2}$. Prog. Theor. Phys. Suppl. 159, 22-32 (2005).

38. Koga, A., Okunishi, K. \& Kawakami, N. First-order quantum phase transition in the orthogonal-dimer spin chain. Phys. Rev. B 62, 5558-5563 (2000). 